\title{
Uniform Approximations of Bernoulli and Euler Polynomials in Terms of Hyperbolic Functions
}

\author{
By José L. López and Nico M. Temme
}

Bernoulli and Euler polynomials are considered for large values of the order. Convergent expansions are obtained for $B_{n}(n z+1 / 2)$ and $E_{n}(n z+1 / 2)$ in powers of $n^{-1}$, and coefficients are rational functions of $z$ and hyperbolic functions of argument $1 /(2 z)$. These expansions are uniformly valid for $|z \pm i / 2 \pi|>1 / 2 \pi$ and $|z \pm i / \pi|>1 / \pi$, respectively. For a real argument, the accuracy of these approximations is restricted to the monotonic region. The range of validity of the uniformity parameter $z$ is enlarged, respectively, to regions of the form $|z \pm i / 2(m+1) \pi|>1 / 2(m+1) \pi$ and $|z \pm i /(2 m+1) \pi|>1 /(2 m+1) \pi, m=1,2,3, \ldots$, by adding certain combinations of incomplete gamma functions to these uniform expansions. In addition, the convergence of these improved expansions is stronger, and for a real argument, the accuracy of these improved approximations is also better in the oscillatory region.

\section{Introduction}

The Bernoulli and Euler polynomials of degree $n$ and complex argument $z$, denoted by $B_{n}(z)$ and $E_{n}(z)$, respectively, are defined by ([1], Eq. 23.1.1.),

$$
\frac{w e^{w z}}{e^{w}-1}=\sum_{n=0}^{\infty} \frac{B_{n}(z)}{n !} w^{n}, \quad|w|<2 \pi,
$$

Address for correspondence: Professor Nico M. Temme, CWI, P.O. Box 94079, 1090 GB Amsterdam, The Netherlands. E-mail: jllopez@posta.unizar.es, nicot@cwi.nl. 


$$
\frac{2 e^{w z}}{e^{w}+1}=\sum_{n=0}^{\infty} \frac{E_{n}(z)}{n !} w^{n}, \quad|w|<\pi .
$$

Standard texts on the classical theory of Bernoulli and Euler polynomials and numbers are, for example, chapter VI in [2] and chapters V and VI in [3]. A very complete bibliography up to 1960 concerning tables and applications of the theory of Bernoulli and Euler polynomials can be found in [4]. Fore more recent works (after 1960), the reader is referred to [5-11] and references therein for a very complete survey of formulas involving these polynomials, zeros, asymptotic behavior, integral representations, and a number of other properties. Nevertheless, an extensive list of formulas involving Bernoulli and Euler polynomials can be found in ([1], pp. 803-806; [12], pp. 35-43; [13], pp. 25-32; [14], pp. 1076-1080; [15], Vol. 3, pp. 55-57; and [16], pp. 2-17).

Here, we are interested in approximations of these polynomials for large order $n$. Convergent expansions in terms of trigonometric functions for real $z$ and $0 \leq z \leq 1$ can be found in ([1], 23.1.16-23.1.18). A more detailed study about these expansions concerned with the type of convergence is investigated in [7], where similar expansions for generalized Bernoulli polynomials are also obtained. In [5], asymptotic expansions of (generalized) Bernoulli polynomials are obtained in terms of elementary functions and also in terms of gamma functions. These expansions happen to fail when the argument $z$ is allowed to grow arbitrarily. Here, we are concerned with finding approximations of the Bernoulli and Euler polynomials for large order $n$ that also remain valid for a large argument $z$. In particular, the purpose of this article is to obtain approximations of $B_{n}(n z+1 / 2)$ and $E_{n}(n z+1 / 2)$ for large $n$ which are uniformly valid in some unbounded region of the complex variable $z$.

\subsection{Summary of results}

Our main results are summarized as follows:

1. For $\left|z \pm \frac{i}{2 \pi}\right|>\frac{1}{2 \pi}, n \geq 1$,

$$
\begin{array}{r}
B_{n}\left(n z+\frac{1}{2}\right)=\frac{n^{n} z^{n-1}}{2 \sinh \left(\frac{1}{2 z}\right)}\left\{1+\left[1+4\left(z-\frac{1}{2} \operatorname{coth}\left(\frac{1}{2 z}\right)\right) \operatorname{coth}\left(\frac{1}{2 z}\right)\right]\right. \\
\left.\times \frac{1}{8 n z^{2}}+\infty\left(\frac{1}{n^{2}}\right)\right\} .
\end{array}
$$

2. For $\left|z \pm \frac{i}{\pi}\right|>\frac{1}{\pi}, n \geq 1$,

$$
E_{n}\left(n z+\frac{1}{2}\right)=\frac{(n z)^{n}}{\cosh \left(\frac{1}{2 z}\right)}\left[1+\left(1-2 \tanh ^{2}\left(\frac{1}{2 z}\right)\right) \frac{1}{8 n z^{2}}+\mathscr{O}\left(\frac{1}{n^{2}}\right)\right] \text {. }
$$


3. For $\left|z \pm \frac{i}{4 \pi}\right|>\frac{1}{4 \pi}, n \geq 1$,

$$
\begin{aligned}
B_{n}\left(n z+\frac{1}{2}\right)=\left[\frac{e^{2 \pi i n z}}{(2 \pi i)^{n}} \Gamma(n+1,2 \pi i n z)+\frac{e^{-2 \pi i n z}}{(-2 \pi i)^{n}} \Gamma(n+1,-2 \pi i n z)\right] \\
+(n z)^{n}\left\{\left[\frac{1}{2 z \sinh \left(\frac{1}{2 z}\right)}-\frac{8 \pi^{2} z^{2}}{1+4 \pi^{2} z^{2}}\right]+\left[\frac{1}{16 z^{3} \sinh \left(\frac{1}{2 z}\right)}\right.\right. \\
\times\left(1+4\left(z-\frac{1}{2} \operatorname{coth}\left(\frac{1}{2 z}\right)\right) \operatorname{coth}\left(\frac{1}{2 z}\right)\right) \\
\left.\left.+\frac{8 \pi^{2} z^{2}\left(3-4 \pi^{2} z^{2}\right)}{\left(1+4 \pi^{2} z^{2}\right)^{3}}\right] \frac{1}{n}+\mathscr{6}\left(\frac{1}{n^{2}}\right)\right\}
\end{aligned}
$$

4. For $\left|z \pm \frac{i}{3 \pi}\right|>\frac{1}{3 \pi}, n \geq 1$,

$$
\begin{aligned}
E_{n}\left(n z+\frac{1}{2}\right)=2 i\left[\frac{e^{\pi i n z}}{(\pi i)^{n+1}} \Gamma(n+1, \pi i n z)-\frac{e^{-\pi i n z}}{(-\pi i)^{n+1}} \Gamma(n+1,-\pi i n z)\right] \\
+(n z)^{n}\left\{\left[\frac{1}{\cosh \left(\frac{1}{2 z}\right)}-\frac{4 \pi z^{2}}{1+\pi^{2} z^{2}}\right]+\left[\frac{4 \pi z^{2}\left(3-\pi^{2} z^{2}\right)}{\left(1+\pi^{2} z^{2}\right)^{3}}\right.\right. \\
\left.+\frac{1}{8 z^{2} \cosh \left(\frac{1}{2 z}\right)}\left(1-2 \tanh ^{2}\left(\frac{1}{2 z}\right)\right)\right] \frac{1}{n} \\
\left.+\mathscr{O}\left(\frac{1}{n^{2}}\right)\right\}
\end{aligned}
$$

For the given $z$-domains, these approximations have the indicated asymptotic properties, and, in addition, they are the first terms of convergent expansions.

\section{Uniform expansions and the saddle point method}

From definitions (1) and (2),

$$
B_{n}\left(z+\frac{1}{2}\right)=\frac{n !}{2 \pi i} \int_{C} \frac{w e^{w z}}{2 \sinh (w / 2)} \frac{d w}{w^{n+1}},
$$

and

$$
E_{n}\left(z+\frac{1}{2}\right)=\frac{n !}{2 \pi i} \int_{C} \frac{e^{w z}}{\cosh (w / 2)} \frac{d w}{w^{n+1}},
$$

where the contour $C$ encircles the origin in the counterclockwise direction and contains no poles of $w \sinh ^{-1}(w / 2)$ or $\cosh ^{-1}(w / 2)$, respectively. The shift of $1 / 2$ in the variable $z$ is introduced to have reflection symmetry $z \rightarrow$ $-z$ in these polynomials. To deal with both $B_{n}(z)$ and $E_{n}(z)$ simultaneously, 
we will start this section with generic polynomials defined by the contour integral

$$
P_{n}(z)=\frac{n !}{2 \pi i} \int_{C} f(w) e^{w z} \frac{d w}{w^{n+1}},
$$

where $f(w)$ is a meromorphic function with simple poles $w_{1}, w_{2}, \ldots$ and analytic at the origin. The contour $C$ is a circle whose center is at the origin and which contains no poles of $f(w)$ inside. Bernoulli and Euler polynomials defined by (3) and (4) are nothing but particular cases.

We can write (5) in the form

$$
P_{n}(n z)=\frac{n !}{2 \pi i} \int_{C} f(w) e^{n(w z-\log (w))} \frac{d w}{w} .
$$

The key observation used for obtaining approximations of $P_{n}(n z)$ for large $n$ and fixed $z$ is the following. The main contribution of the integrand above to the integral originates at the saddle point of the argument of the exponential [17], that is, at the point $w=z^{-1}$. Approximations of $P_{n}(n z)$ for large $n$ and fixed $z$ can be obtained by expanding $f(w)$ around the saddle point [18-21]. Therefore, if $z^{-1}$ is not a pole of $f(w)$, we expand

$$
f(w)=\sum_{k=0}^{\infty} \frac{f^{(k)}\left(z^{-1}\right)}{k !}\left(w-z^{-1}\right)^{k}, \quad\left|w-z^{-1}\right|<r
$$

where $r$ is the distance from $z^{-1}$ to the nearest singularity of $f(w)$. The radius $\epsilon_{1}$ of the contour $C$ in definition (5) of $P_{n}(z)$ can be chosen as close to zero as necessary. Then, for $w \in C\left(|w|=\epsilon_{1}\right)$, the above series is absolutely convergent if $\left|z^{-1}\right|<\left|z^{-1}-w_{k}\right| \forall k=1,2, \ldots$ (see Figure 1(a) where the particular case $f(w)=(w / 2) \sinh (w / 2)$ is represented).

Substituting expansion (6) in (5), we obtain

$$
P_{n}(n z)=\frac{n !}{2 \pi i} \int_{C} \sum_{k=0}^{\infty} \frac{f^{(k)}\left(z^{-1}\right)}{k !}\left(w-z^{-1}\right)^{k} e^{n w z} \frac{d w}{w^{n+1}} .
$$

The $k$ th derivative of $f(w)$ evaluated at point $z^{-1}$ may be written

$$
f^{(k)}\left(z^{-1}\right)=\frac{k !}{2 \pi i} \int_{C^{\prime}} \frac{f(t) d t}{\left(t-z^{-1}\right)^{k+1}},
$$

where the contour $C^{\prime}$ is a circle around the point $z^{-1}$ whose radius $R \equiv$ $\left|t-z^{-1}\right|$ is smaller than the distance from $z^{-1}$ to any of the singularities $w_{k}$ of $f(t)$, that is, $R \equiv \min _{k \in N}\left|w_{k}-z^{-1}\right|-\epsilon_{2}$ for some $\epsilon_{2}>0$ (see Figure 2(b) where the particular case $w_{k}= \pm 2 k \pi i$ is represented). Therefore,

$$
\left|f^{(k)}\left(z^{-1}\right)\right| \leq K \frac{k !}{R^{k}}
$$




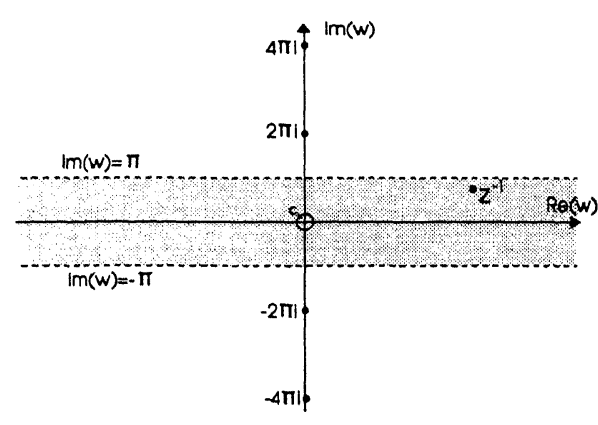

(a)

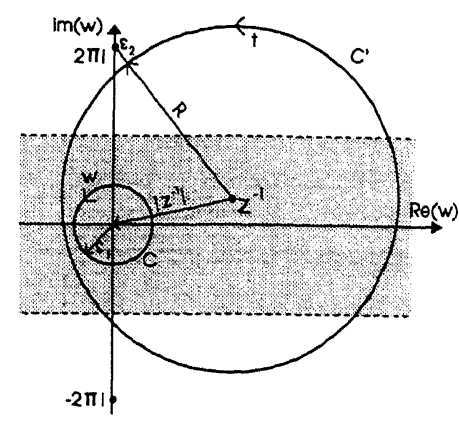

(b)

Figure 1. (a) The meromorphic function $f(w)=(w / 2) \sinh (w / 2)$ has singularities $w_{k}=$ $\pm 2 k \pi i, k=1,2, \ldots$. For small $\epsilon_{1}$, the validity of (6) is guaranteed if the saddle point $z^{-1}$ is closer to the origin than to any of the singularities $w_{k}$. This happens for $z^{-1}$ inside a strip of width $2 \pi$ along the real axis. (b) For $z^{-1}$ inside that strip, we can choose small enough $\epsilon_{1}$ and $\epsilon_{2}=\left|z^{-1}-2 \pi i\right|-R$ such that the circle $C$ is inside the circle $C^{\prime}$. Then, $a \equiv \max _{w \in C} \mid w-$ $z^{-1} \mid / R<1$ and (9) holds.

where $K$ does not depend on $k$. Then, for $\left|z^{-1}\right|<\left|z^{-1}-w_{k}\right| \forall k=1,2, \ldots$ and small enough $\epsilon_{1}$ and $\epsilon_{2}, \max _{w \in C}\left|w-z^{-1}\right| / R \equiv a<1$ for a certain $a>0$ which only depends on $z, \epsilon_{1}$, and $R$ (see Figure 1(b)). Taking the modulus in (7) and using (8), we obtain

$$
\left|P_{n}(n z)\right| \leq K_{n} \sum_{k=0}^{\infty} a^{k} \int_{C}\left|e^{n w z} \frac{d w}{w^{n+1}}\right|<\infty
$$

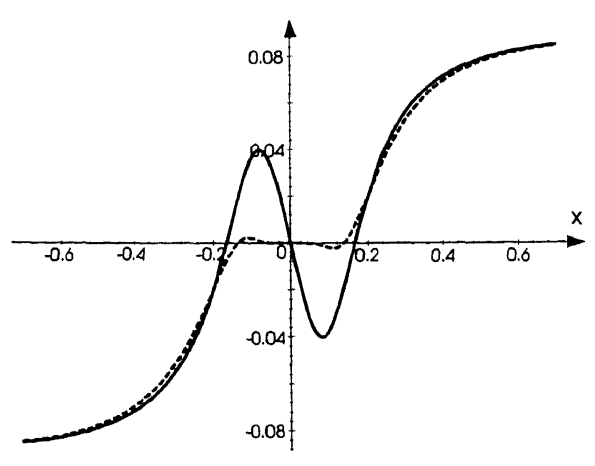

(a) $n=3$.

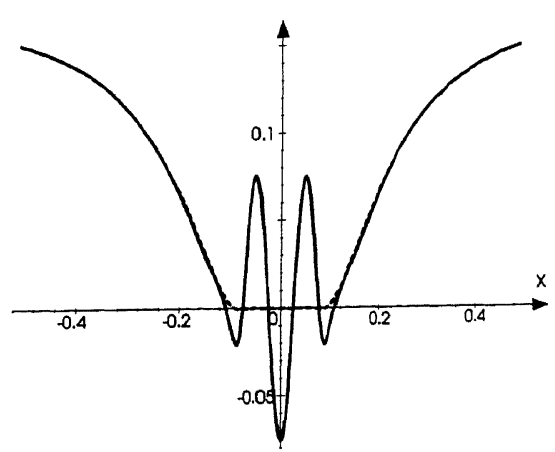

(b) $n=10$.

Figure 2. Solid lines represent $B_{n}(n x+1 / 2)$ for several values of $n$, whereas dashed line represent the right-hand side of (19) with $z \equiv x$, both normalized by the factor $\left(1+|x / a|^{n}\right)^{-1}$ where $a$ is half the width of the oscillatory region. 
where $K_{n}$ is a certain constant. Therefore, after substituting (6) in (5), we can apply Fubini's theorem and interchange summation and integration to obtain, for $\left|z^{-1}\right|<\left|z^{-1}-w_{i}\right| \forall i=1,2,3, \ldots$,

$$
P_{n}(n z)=(n z)^{n} \sum_{k=0}^{\infty} \frac{f^{(k)}\left(z^{-1}\right)}{k !} \Phi_{k}(n, z),
$$

where

$$
\Phi_{k}(n, z)=\frac{1}{2 \pi i} \frac{n !}{(n z)^{n}} \int_{C}\left(w-z^{-1}\right)^{k} e^{n z w} \frac{d w}{w^{n+1}} .
$$

The functions $\Phi_{k}(n, z)$ are polynomials in $n$ divided by powers of $z$ and constitute an asymptotic sequence for $n \rightarrow \infty$. More precisely,

LEMMA 1. The functions $\Phi_{k}(n, z)$ defined in (11) can be represented in the form

$$
\Phi_{k}(n, z)=\frac{p_{k}(n)}{(n z)^{k}}
$$

where

$$
p_{0}(n)=1, \quad p_{1}(n)=0, \quad p_{2}(n)=-n, \quad p_{3}(n)=2 n,
$$

and the remaining polynomials $p_{k}(n)$ are given by the recurrence

$$
p_{k}(n)=(1-k)\left(p_{k-1}(n)+n p_{k-2}(n)\right) .
$$

Proof. By direct calculation from definition (11) for $k=0$ and $k=1$, we obtain $\Phi_{0}(n, z)=1$ and $\Phi_{1}(n, z)=0$. For $k \geq 2$, we can write

$$
\Phi_{k}(n, z)=\frac{1}{2 \pi i} \frac{n !}{(n z)^{n+1}} \int_{C}\left(w-z^{-1}\right)^{k-1} \frac{d}{d w}\left\{e^{n z\left[w-z^{-1} \ln (w)\right]}\right\} d w .
$$

Integrating by parts and after straightforward operations, we obtain

$$
\Phi_{k}(n, z)=\frac{1-k}{n z}\left[z^{-1} \Phi_{k-2}(n, z)+\Phi_{k-1}(n, z)\right]
$$

from which (14) follows trivially.

LEMMA 2. For fixed $z \neq 0$, the sequence $\Phi_{k}(n, z)$ is an asymptotic sequence for $n \rightarrow \infty$ that satisfies $\Phi_{k}(n, z)=\mathscr{O}\left(n^{[k / 2]-k}\right)$.

Proof. It is trivially verified for $\Phi_{0}(n, z)$ and $\Phi_{1}(n, z)$. For $k \geq 2$ it can be easily proved by induction over $k$ by using (12) and the recurrence (14). 
We can summarize this discussion as follows:

THEOREM 1. The polynomials $P_{n}(n z)$ defined in (5), where $f(w)$ is a meromorphic function with simple singularities $w_{1}, w_{2}, \ldots$ and analytic in the origin, may be expanded as the infinite sum

$$
P_{n}(n z)=(n z)^{n} \sum_{k=0}^{\infty} \frac{f^{(k)}\left(z^{-1}\right)}{k !} \frac{p_{k}(n)}{(n z)^{k}}
$$

valid for $z \in \mathbb{C},\left|z^{-1}\right|<\left|z^{-1}-w_{i}\right| \forall i=1,2,3, \ldots$, where $p_{k}(n)$ are the polynomials defined in (13)-(14).

Remark 1. Observe that the polynomials $p_{k}(n)$ that appear in the expansion (16) of $P_{n}(n z)$ are the same for any set of polynomials of the family $P_{n}(z)$ defined in (5). The particular information about the particular set of polynomials defined by each $f(w)$ in $(5)$ is contained in the coefficients $f^{(k)}\left(z^{-1}\right)$.

\subsection{Uniform expansions of Bernoulli polynomials}

The Bernoulli polynomials $B_{n}(n z+1 / 2)$ may be expanded in the form (16) with $f(w)=(w / 2) / \sinh (w / 2)$ and $w_{k}= \pm 2 k \pi i, k=1,2,3, \ldots$ In this case, for $w \in C$, Equation (6) reads,

$$
\frac{\frac{w}{2}}{\sinh \left(\frac{w}{2}\right)}=\sum_{k=0}^{\infty} \frac{f^{(k)}\left(z^{-1}\right)}{k !}\left(w-z^{-1}\right)^{k}, \quad\left|w-z^{-1}\right|<\left| \pm 2 \pi i-z^{-1}\right| .
$$

After straightforward operations, we find that the derivatives $f^{(k)}\left(z^{-1}\right)$ are given by the recurrence

$$
\begin{aligned}
f^{(k)}\left(z^{-1}\right)= & -\sum_{l=1}^{\lfloor k / 2\rfloor}\left(\begin{array}{c}
k \\
2 l
\end{array}\right) \frac{f^{k-2 l}\left(z^{-1}\right)}{4^{l}} \\
& -\frac{1}{2} \operatorname{coth}\left(\frac{1}{2 z}\right) \sum_{l=0}^{\lfloor(k-1) / 2\rfloor}\left(\begin{array}{c}
k \\
2 l+1
\end{array}\right) \frac{f^{k-2 l-1}\left(z^{-1}\right)}{4^{l}}, \quad k \geq 2,
\end{aligned}
$$

where

$$
f^{(0)}\left(z^{-1}\right)=\frac{1}{2 z \sinh \left(\frac{1}{2 z}\right)} \quad \text { and } \quad f^{(1)}\left(z^{-1}\right)=\frac{z-\frac{1}{2} \operatorname{coth}\left(\frac{1}{2 z}\right)}{2 z \sinh \left(\frac{1}{2 z}\right)}
$$

In the results shown we also use $f^{(2)}\left(z^{-1}\right)$, which is given by

$$
f^{(2)}\left(z^{-1}\right)=-\frac{1}{8 z \sinh \left(\frac{1}{2 z}\right)}\left\{1+4\left[z-\frac{1}{2} \operatorname{coth}\left(\frac{1}{2 z}\right)\right] \operatorname{coth}\left(\frac{1}{2 z}\right)\right\} \text {. }
$$

Introducing these $f^{(k)}\left(z^{-1}\right)$ in (16) and retaining only the first three terms of the expansion for convenience, we obtain the following corollary: 
COROllaRY 1. For $\left|\operatorname{Im}\left(z^{-1}\right)\right|<\pi\left(\right.$ or $\left.\left|z \pm \frac{i}{2 \pi}\right|>\frac{1}{2 \pi}\right)$ and $n \geq 1$

$$
\begin{aligned}
B_{n}\left(n z+\frac{1}{2}\right)= & \frac{n^{n} z^{n-1}}{2 \sinh \left(\frac{1}{2 z}\right)} \\
& \times\left\{1+\left[1+4\left(z-\frac{1}{2} \operatorname{coth}\left(\frac{1}{2 z}\right)\right) \operatorname{coth}\left(\frac{1}{2 z}\right)\right]\right. \\
& \left.\times \frac{1}{8 n z^{2}}+\mathscr{\odot}\left(\frac{1}{n^{2}}\right)\right\} .
\end{aligned}
$$

Figure 2 shows the accuracy of approximation (19) for several values of $n$ for real values of the uniformity parameter $z$.

Although expansion (16) with $f^{(k)}\left(z^{-1}\right)$ given by (18) is convergent for $\left|z \pm \frac{i}{2 \pi}\right|>\frac{1}{2 \pi}$, convergence is slow for $|z| \leq \pi^{-1}$ (and $\left|z \pm \frac{i}{2 \pi}\right|>\frac{1}{2 \pi}$ ) and quite fast for $|z|>\pi^{-1}$. The relative error decreases for increasing $|z|$ or $n$. For example, for $|z| \sim \pi^{-1}$ and $n=10$, the relative error is $\sim 10^{-2}$. For $n=40$ and $|z| \sim 1$, the relative error is $\sim 10^{-5}$. The accuracy is even better for a real argument, as shown in Figure 2. For a real argument, the oscillatory region of $B_{n}(n x+1 / 2)$ is contained in $|x| \leq \pi^{-1}$, whereas the monotonic region contains $|x|>\pi^{-1}$. Therefore, for a real argument, the accuracy of approximation (19) is restricted to the monotonic region.

\subsection{Uniform expansions of Euler polynomials}

The Euler polynomials $E_{n}(n z+1 / 2)$ may be expanded in the form (16) with $f(w)=1 / \cosh (w / 2)$ and $w_{j}= \pm(2 j+1) \pi i, j=0,1,2, \ldots$ Calculations similar to those of the above subsection may be performed in this case. For $w \in C$, Equation (6) reads

$$
\frac{1}{\cosh (w / 2)}=\sum_{k=0}^{\infty} \frac{f^{(k)}\left(z^{-1}\right)}{k !}\left(w-z^{-1}\right)^{k}, \quad\left|w-z^{-1}\right|<\left| \pm \pi i-z^{-1}\right| .
$$

After straightforward operations, we find that the derivatives $f^{(k)}\left(z^{-1}\right)$ are given by the recurrence

$$
\begin{aligned}
f^{(k)}\left(z^{-1}\right)= & -\sum_{l=1}^{\lfloor k / 2\rfloor}\left(\begin{array}{c}
k \\
2 l
\end{array}\right) \frac{f^{k-2 l}\left(z^{-1}\right)}{4^{l}} \\
& -\frac{1}{2} \tanh \left(\frac{1}{2 z}\right) \sum_{l=0}^{\lfloor(k-1) / 2\rfloor}\left(\begin{array}{c}
k \\
2 l+1
\end{array}\right) \frac{f^{k-2 l-1}\left(z^{-1}\right)}{4^{l}}, \quad k \geq 1,
\end{aligned}
$$

where

$$
f^{(0)}\left(z^{-1}\right)=\frac{1}{\cosh \left(\frac{1}{2 z}\right)}
$$




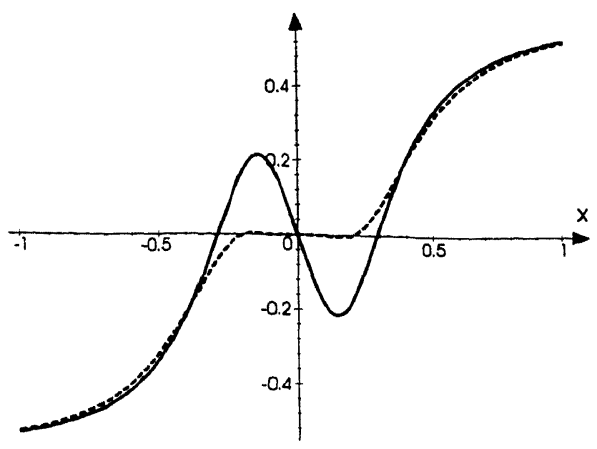

(a) $n=3$.

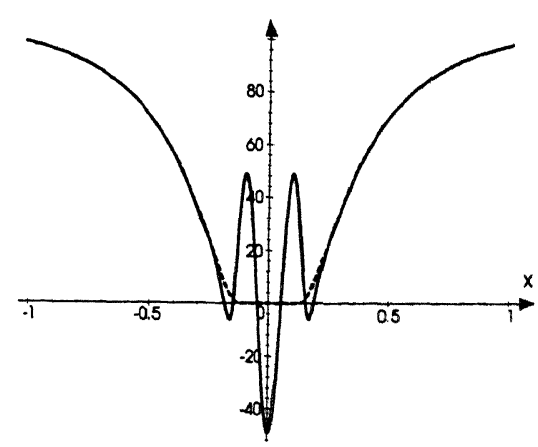

(b) $n=10$.

Figure 3. Solid lines represent $E_{n}(n x+1 / 2)$ for several values of $n$, whereas dashed lines represent the right-hand side of (22) with $z \equiv x$, both normalized by the same factor as in Figure 2.

In the results shown, we also use $f^{(2)}\left(z^{-1}\right)$, which is given by

$$
f^{(2)}\left(z^{-1}\right)=\frac{1}{4 \cosh \left(\frac{1}{2 z}\right)}\left[2 \tanh ^{2}\left(\frac{1}{2 z}\right)-1\right] \text {. }
$$

Introducing these $f^{(k)}\left(z^{-1}\right)$ in (16) and retaining for convenience only the first three terms of the expansion, we obtain the following corollary:

COROLlary 2. For $\left|\operatorname{Im}\left(z^{-1}\right)\right|<\frac{\pi}{2}$ (or $\left|z \pm \frac{i}{\pi}\right|>\frac{1}{\pi}$ ) and $n \geq 1$,

$$
E_{n}\left(n z+\frac{1}{2}\right)=\frac{(n z)^{n}}{\cosh \left(\frac{1}{2 z}\right)}\left\{1+\left[1-2 \tanh ^{2}\left(\frac{1}{2 z}\right)\right] \frac{1}{8 n z^{2}}+c\left(\frac{1}{n^{2}}\right)\right\} \text {. }
$$

Figure 3 shows the accuracy of approximation (22) for several values of $n$ for real values of the uniformity parameter $z$.

As in the Bernoulli case, although expansion (16) with $f^{(k)}\left(z^{-1}\right)$ given by (22) is convergent for $\left|z \pm \frac{i}{\pi}\right|>\frac{1}{\pi}$, convergence is slow for $|z| \leq 2 \pi^{-1}$ (and $\left|z \pm \frac{i}{\pi}\right|>\frac{1}{\pi}$ ) and quite fast for $|z|>2 \pi^{-1}$. The relative error decreases for increasing $|z|$ or $n$. For example, for $|z| \sim 2 \pi^{-1}$ and $n=10$, the relative error is $\sim 10^{-2}$. For $n=40$ and $|z| \sim 1$, the relative error is $\sim 10^{-4}$. The accuracy is even better for a real argument, as shown in Figure 3. For a real argument, the oscillatory region of $E_{n}(n x+1 / 2)$ is also contained in $|x| \leq 2 \pi^{-1}$, whereas the monotonic region contains $|x|>2 \pi^{-1}$. Therefore, the accuracy of approximation (22) is restricted to the monotonic region.

\section{Enlarging the region of validity of the uniformity parameter $z$}

In Theorem 1 we proved that the validity of approximation (16) is restricted to the region $\left|z^{-1}\right|<\left|z^{-1}-w_{i}\right| \forall i=1,2,3, \ldots$ (in the particular cases of 
the Bernoulli and Euler polynomials, the uniformity parameter $z$ is restricted to be outside the discs $\left|z \pm \frac{i}{2 \pi}\right| \leq \frac{1}{2 \pi}$ and $\left|z \pm \frac{i}{\pi}\right| \leq \frac{1}{\pi}$, respectively). The expansions in these $z$-domains are convergent series, as in (16) we conclude from computer experiments (see also Figures 2 and 3 ) that, in addition, in the Bernoulli and Euler cases, the uniformity parameter should satisfy $|z| \geq \pi^{-1}$ and $|z| \geq 2 \pi^{-1}$, respectively, to yield a good approximation by using only the first few terms of the expansion (16).

However, the region $\left\{z \in \mathbb{C},\left|z^{-1}\right|<\left|z^{-1}-w_{i}\right| \forall i=1,2,3, \ldots\right\}$ may be enlarged by "isolating" the contribution of the poles $w_{1}, w_{2}, \ldots$ of $f(w)$. Take the (simple) poles $\left\{w_{i} \in \mathbb{C}, i=1,2,3, \ldots\right\}$ of $f(w)$, ordered by increasing modulus $\left|w_{i}\right| \leq\left|w_{i+1}\right|$ and define

$$
f_{m}(w)=f(w)-\sum_{k=1}^{m} \frac{r_{k}}{w-w_{k}},
$$

where $r_{k}$ are the residues of $f(w)$ in $w_{k}$. The function $f_{m}(w)$ has no poles inside the disc $\left\{w \in \mathbb{C},|w|<\left|w_{m+1}\right|\right\}$. Introducing (23) in (5), we obtain

$$
P_{n}(z)=P_{n}^{m}(z)+Q_{n}^{m}(z)
$$

where

$$
P_{n}^{m}(z)=\frac{n !}{2 \pi i} \int_{C} f_{m}(w) e^{w z} \frac{d w}{w^{n+1}}
$$

and

$$
Q_{n}^{m}(z)=\frac{n !}{2 \pi i} \sum_{k=1}^{m} r_{k} \int_{C} \frac{e^{w z}}{w-w_{k}} \frac{d w}{w^{n+1}} .
$$

Now, the poles $w_{m+1}, w_{m+2}, \ldots$ of the function $f_{m}(w)$ are farther away from the origin than the poles of the function $f(w)$. Repeating the process of the last section for $P_{n}^{m}(z)$ instead of $P_{n}(z)$, we obtain

$$
P_{n}^{m}(n z)=(n z)^{n} \sum_{k=0}^{\infty} \frac{f^{(k)}\left(z^{-1}\right)+h_{m}^{(k)}\left(z^{-1}\right)}{k !} \frac{p_{k}(n)}{(n z)^{k}}
$$

that is valid for $\left|z^{-1}\right|<\left|z^{-1}-w_{i}\right| \forall i=m+1, m+2, \ldots$ (and $z \neq 0$ ), where $h_{m}^{(k)}(w)$ is the $k$ th derivative of

$$
h_{m}(w)=-\sum_{l=1}^{m} \frac{r_{l}}{w-w_{l}} .
$$

Therefore, now the range of validity of expansion (27) is larger than that of expansion (16). On the other hand, the functions $Q_{n}^{m}(z)$ defined in (26) are 
just combinations of incomplete gamma functions. By shifting the integration contour by $w=w_{k}+t$ in each integral in (26), writing

$$
\frac{e^{t z}}{t}=\int_{0}^{z} e^{t x} d x+\frac{1}{t}
$$

and after straightforward operations using elementary properties of incomplete gamma functions ([16], Chap. 11, Section 2), we obtain

$$
\frac{n !}{2 \pi i} \int_{C} \frac{e^{w z}}{w-w_{k}} \frac{d w}{w^{n+1}}=e^{w_{k} z}\left[\int_{0}^{z} e^{-w_{k} t} t^{n} d t-\frac{n !}{w_{k}^{n+1}}\right]=-\frac{e^{w_{k} z}}{w_{k}^{n+1}} \Gamma\left(n+1, w_{k} z\right),
$$

that is simply $-n ! / w_{k}^{n+1}$ multiplied by the Taylor polynomial of degree $n$ in $z=0$ of $e^{w_{k} z}$. Therefore,

$$
Q_{n}^{m}(z)=-\sum_{k=1}^{m} \frac{r_{k} e^{w_{k} z}}{w_{k}^{n+1}} \Gamma\left(n+1, w_{k} z\right)
$$

and we have the following:

THEOREM 2. The polynomials $P_{n}(z)$ defined in (5) by a meromorphic function $f(w)$ analytic in the origin with simple poles $w_{1}, w_{2}, \ldots$ (and respective residues $\left.r_{1}, r_{2}, \ldots\right)$, can be represented, for each integer $m>0$, as

$$
\begin{aligned}
P_{n}(n z)= & -\sum_{k=1}^{m} \frac{r_{k} e^{w_{k} n z}}{w_{k}^{n+1}} \Gamma\left(n+1, w_{k} n z\right) \\
& +(n z)^{n} \sum_{k=0}^{\infty} \frac{f^{(k)}\left(z^{-1}\right)+h_{m}^{(k)}\left(z^{-1}\right)}{k !} \frac{p_{k}(n)}{(n z)^{k}}
\end{aligned}
$$

that is valid for $z \in \mathbb{C},\left|z^{-1}\right|<\left|z^{-1}-w_{i}\right|, \forall i=m+1, m+2, \ldots$, where the polynomials $p_{k}(n)$ are given in (14) and $h_{m}^{(k)}\left(z^{-1}\right)$ is the kth derivative of the function $h_{m}(w)$ defined in (28). Each term of the finite sum in the first line in the above equation equals $n ! r_{k} / w_{k}^{n+1}$ multiplied by the Taylor polynomial of degree $n$ in $z=0$ of $e^{w_{k} n z}$.

The "isolation" technique used above for enlarging the region of validity of $z$ is quite similar to that employed to obtain uniform asymptotic expansions of contour integrals with a saddle point near a pole ([17], Chap. 7, Section 2). There, by a change of variable, the contribution of the poles (Equation (26)) is expressed as an error function. Here, it is expressed just as a combination of incomplete gamma functions.

The series expansion of $P_{n}^{m}(n z)$ on the right-hand side of (27) converges in a larger region $\left|z^{-1}\right|<\left|z^{-1}-w_{i}\right| \forall i=m+1, m+2, \ldots$ But, moreover, the polynomial $Q_{n}^{m}(z)$ has been defined in (26) by using a function $-h_{m}(w)$ 
in the integral that has the same first $2 m$ poles and the same residues as the function $f(w)$ that defines $P_{n}(z)$ in (5). The contour $C$ in (5) and in (26) may be chosen to pass near the singularities of $f(w)$ closest to the origin. If $|z|$ is small, the saddle point is far away from the path $C$ and the greatest contribution to the integral is given by the piece of integral closest to these singularities. Therefore both integrals in (5) and in (26) are dominated by the same singularity, and then these integrals should be similar, that is, $P_{n}(n z) \simeq Q_{n}^{m}(n z)$ for small $|z|$ and $m \geq 1$. Therefore, for small $|z|$, it must happen that $\left|P_{n}^{m}(n z)\right| \ll\left|Q_{n}^{m}(n z)\right|$. We check these facts in the Bernoulli and Euler examples.

\subsection{Bernoulli polynomials}

The first $2 m$ poles and residues of $f(w)=(w / 2) / \sinh (w / 2)$ are $w_{k}= \pm 2 k \pi i$, $r_{k}=(-1)^{k} w_{k}, k=1,2, \ldots, m$. From (28) and after straightforward algebra, one may easily check that the derivatives $h_{m}^{(k)}\left(z^{-1}\right)$ of $h_{m}(w)$ at the saddle point $z^{-1}$ can be obtained from

$$
h_{m}^{(k)}\left(z^{-1}\right)=\sum_{l=1}^{m}(-1)^{l} h_{l}^{(k)}
$$

where $h_{l}^{(k)}$ are given by the recursive formula

$$
h_{l}^{(k)}=-\frac{k}{z^{-2}+4 \pi^{2} l^{2}}\left[2 z^{-1} h_{l}^{(k-1)}+(k-1) h_{l}^{(k-2)}\right]
$$

for $k \geq 2$, where

$$
h_{l}^{(0)}=\frac{8 \pi^{2} l^{2}}{z^{-2}+4 \pi^{2} l^{2}} \quad \text { and } \quad h_{l}^{(1)}=-\frac{16 \pi^{2} l^{2}}{z\left(z^{-2}+4 \pi^{2} l^{2}\right)^{2}} .
$$

In the results shown, we also use $h_{l}^{(2)}$, which is given by

$$
h_{l}^{(2)}=\frac{16 \pi^{2} l^{2}\left(3 z^{-2}-4 l^{2} \pi^{2}\right)}{\left(z^{-2}+4 l^{2} \pi^{2}\right)^{3}} \text {. }
$$

Introducing $f^{(k)}\left(z^{-1}\right)$ given in (18) and $h_{m}^{(k)}\left(z^{-1}\right)$ given in (30)-(31) in Equation (29), using $w_{k}= \pm 2 k \pi i, r_{k}=(-1)^{k} w_{k}, k=1,2, \ldots, m$, and retaining only the first three terms of the infinite sum in the second line of (29), we obtain the following corollary: 
COROllary 3. For $\left|\operatorname{Im}\left(z^{-1}\right)\right|<(m+1) \pi\left(\right.$ or $\left.\left|z \pm \frac{i}{2(m+1) \pi}\right|>\frac{1}{2(m+1) \pi}\right)$ and $n, m \geq 1$,

$$
\begin{aligned}
B_{n}\left(n z+\frac{1}{2}\right)=- & \sum_{k=1}^{m}(-1)^{k}\left[\frac{e^{2 k \pi i n z}}{(2 k \pi i)^{n}} \Gamma(n+1,2 k \pi i n z)\right. \\
& \left.+\frac{e^{-2 k \pi i n z}}{(-2 k \pi i)^{n}} \Gamma(n+1,-2 k \pi i n z)\right] \\
+ & (n z)^{n}\left\{\left[\frac{1}{2 z \sinh \left(\frac{1}{2 z}\right)}+8 \pi^{2} \sum_{k=1}^{m} \frac{(-1)^{k} k^{2} z^{2}}{1+4 k^{2} \pi^{2} z^{2}}\right]\right. \\
+ & {\left[\frac{1}{16 z^{3} \sinh \left(\frac{1}{2 z}\right)}\left(1+4\left(z-\frac{1}{2} \operatorname{coth}\left(\frac{1}{2 z}\right)\right) \operatorname{coth}\left(\frac{1}{2 z}\right)\right)\right.} \\
& \left.\left.-8 \pi^{2} \sum_{k=1}^{m} \frac{(-1)^{k} k^{2} z^{2}\left(3-4 k^{2} \pi^{2} z^{2}\right)}{\left(1+4 k^{2} \pi^{2} z^{2}\right)^{3}}\right] \frac{1}{n}+\mathscr{O}\left(\frac{1}{n^{2}}\right)\right\} .
\end{aligned}
$$

Remark 2. For $m=1$, by using

$$
\Gamma(n+1, z)=n ! e^{-z} \sum_{j=0}^{n} \frac{z^{j}}{j !},
$$

it follows that the first sum on the right-hand side, that is, the polynomial $Q_{n}^{1}(n z)$, reduces to

$$
Q_{n}^{1}(z)=2 n ! z^{n} \sum_{k=0}^{\lfloor n / 2\rfloor} \frac{(-1)^{k}}{\left(4 \pi^{2} z^{2}\right)^{k}(n-2 k) !} .
$$

This is simply $2 n ! /(2 \pi)^{n}$ multiplied by the Taylor polynomial of degree $n$ in $z=0$ of $\cos (2 \pi n z-\pi n / 2)$, which in turn is the first term of the Fourier expansion of $B_{n}(n z+1 / 2)([1], 23.1 .16)$, which converges very rapidly if $n$ is large.

Figure 4 shows the strong accuracy of approximation (32) already obtained from $n=3$ with $m=1$ for real values of the uniformity parameter $z$.

Approximation (32) is not valid for $\left|z \pm \frac{i}{2(m+1) \pi}\right| \leq \frac{1}{2(m+1) \pi}$. Convergence is slow in the vicinity of these discs and grows sharply for increasing distance from $z$ to these discs and/or increasing $n$. For example, for $m=1$ and $n=10$, the relative error is $\sim 10^{-2}$ for $\left|z \pm \frac{i}{4 \pi}\right| \sim \frac{1}{4 \pi}$ and $\sim 10^{-5}$ for $|z| \sim 1$. For $m=1$ and $n=40$, the relative error is $\sim 10^{-3}$ for $\left|z \pm \frac{i}{4 \pi}\right| \sim \frac{1}{4 \pi}$ and $\sim 10^{-6}$ for $|z| \sim 1$. Accuracy is even better for a real argument, as shown in Figure 4, and differing from approximation (19), accuracy is also good in the oscillatory region. For a real argument and in the oscillatory region, $Q_{n}^{1}(n x) \simeq B_{n}(n x+1 / 2)$ and $P_{n}^{1}(n x)$ tends to zero exponentially fast. On the other hand, the approximation of $B_{n}(n x+1 / 2)$ in the monotonic region given by $P_{n}^{1}(n x)+Q_{n}^{1}(n x)$ is better than the approximation given by $P_{n}(n x)$. 


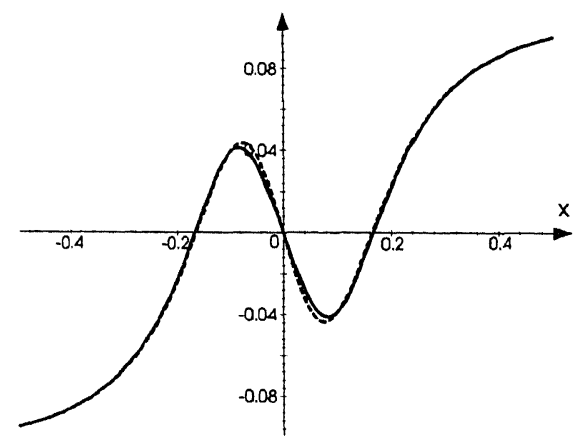

(a) $n=3$.

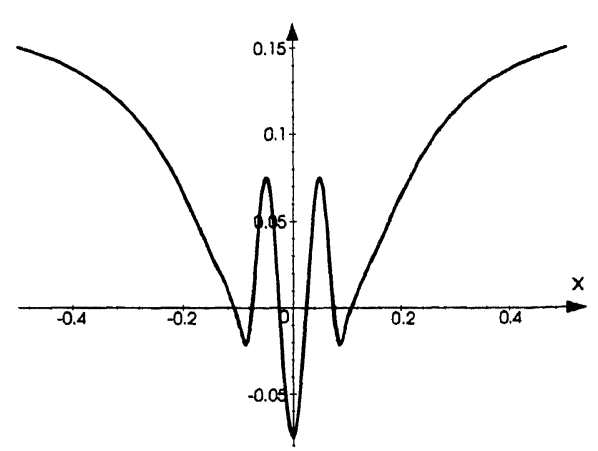

(b) $n=10$.

Figure 4. Solid lines represent $B_{n}(n x+1 / 2)$ for several values of $n$, whereas dashed lines represent the right-hand side of (32) with $z \equiv x$, both normalized by the same factor as in Figure 2.

\subsection{Euler polynomials}

The first $2 m$ poles and residues of $f(w)=1 / \cosh (w / 2)$ are, respectively, $w_{k}= \pm(2 k+1) \pi i$ and $r_{k}=\mp 2 i(-1)^{k}, k=0,1,2, \ldots, m-1$. From (28) and after straightforward algebra, one may easily check that the derivatives $h_{m}^{(k)}\left(z^{-1}\right)$ of $h_{m}(w)$ at the saddle point $z^{-1}$ can be obtained from

$$
h_{m}^{(k)}\left(z^{-1}\right)=\sum_{l=0}^{m-1}(-1)^{l} h_{l}^{(k)}
$$

where $h_{l}^{(k)}$ are given by the recursive formula

$$
h_{l}^{(k)}=-\frac{k}{z^{-2}+(2 l+1)^{2} \pi^{2}}\left[2 z^{-1} h_{l}^{(k-1)}+(k-1) h_{l}^{(k-2)}\right]
$$

that is valid for $k \geq 2$ where

$$
h_{l}^{(0)}=-\frac{4 \pi(2 l+1)}{z^{-2}+(2 l+1)^{2} \pi^{2}} \quad \text { and } \quad h_{l}^{(1)}=\frac{8 \pi(2 l+1) z^{-1}}{\left(z^{-2}+(2 l+1)^{2} \pi^{2}\right)^{2}} .
$$

In the results shown, we also use $h_{l}^{(2)}$, which is given by

$$
h_{l}^{(2)}=-8(2 l+1) \pi \frac{\left(3 z^{-2}-(2 l+1)^{2} \pi^{2}\right)}{\left(z^{-2}+(2 l+1)^{2} \pi^{2}\right)^{3}} .
$$

Introducing $f^{(k)}\left(z^{-1}\right)$ given in Equation (21) and $h_{m}^{(k)}\left(z^{-1}\right)$ given in (33)-(34) in Equation (29), using $w_{k}= \pm(2 k+1) \pi i$ and $r_{k}=\mp 2 i, k=0,1,2, \ldots, m-$ 1 , and retaining only the first three terms of the infinite sum in the second line of (29), we obtain the following corollary: 
COROLLARY 4. For $\left|\operatorname{Im}\left(z^{-1}\right)\right|<(2 m+1) \pi / 2\left(\right.$ or $\left.\left|z \pm \frac{i}{(2 m+1) \pi}\right|>\frac{1}{(2 m+1) \pi}\right)$
and $n, m>1$

$$
\begin{aligned}
& E_{n}\left(n z+\frac{1}{2}\right)=2 i \sum_{k=0}^{m-1}(-1)^{k}\left\{\frac{e^{(2 k+1) \pi i n z}}{[(2 k+1) \pi i]^{n+1}} \Gamma[n+1,(2 k+1) \pi i n z]\right. \\
& \left.-\frac{e^{-(2 k+1) \pi i n z}}{[-(2 k+1) \pi i]^{n+1}} \Gamma[n+1,-(2 k+1) \pi i n z]\right\} \\
& +(n z)^{n}\left\{\left[\frac{1}{\cosh \left(\frac{1}{2 z}\right)}-4 \pi \sum_{k=0}^{m-1} \frac{(-1)^{k}(2 k+1) z^{2}}{1+(2 k+1)^{2} \pi^{2} z^{2}}\right]\right. \\
& +\left[4 \pi \sum_{k=0}^{m-1} \frac{(-1)^{k}(2 k+1) z^{2}\left(3-(2 k+1)^{2} \pi^{2} z^{2}\right)}{\left(1+(2 k+1)^{2} \pi^{2} z^{2}\right)^{3}}\right. \\
& \left.+\frac{1}{8 z^{2} \cosh \left(\frac{1}{2 z}\right)}\left(1-2 \tanh ^{2}\left(\frac{1}{2 z}\right)\right)\right] \frac{1}{n} \\
& \left.+\mathscr{O}\left(\frac{1}{n^{2}}\right)\right\} \text {. }
\end{aligned}
$$

Remark 3. For $m=1$, the first line on the right-hand side, that is, the polynomial $Q_{n}^{1}(n z)$, is simply $4 n ! / \pi^{n+1}$ multiplied by the Taylor polynomial of degree $n$ in $z=0$ of $\cos (\pi n z-\pi n / 2)$, the first term of the Fourier expansion of $E_{n}(n z+1 / 2)([1], 23.1 .16)$,

$$
Q_{n}^{1}(z)=\frac{4 n ! z^{n}}{\pi} \sum_{k=0}^{\lfloor n / 2\rfloor} \frac{(-1)^{k}}{\left(\pi^{2} z^{2}\right)^{k}(n-2 k) !}
$$

Figure 5 shows the strong accuracy of approximation (35) already obtained from $n=3$ with $m=1$ for real values of the uniformity parameter $z$.

Approximation (35) is not valid for $\left|z \pm \frac{i}{(2 m+1) \pi}\right| \leq \frac{1}{(2 m+1) \pi}$. Convergence is slow in the vicinity of these discs and grows sharply for increasing distance from $z$ to these discs and/or increasing $n$. For example, for $m=1$ and $n=10$, the relative error is $\sim 10^{-3}$ for $\left|z \pm \frac{i}{3 \pi}\right| \sim \frac{1}{3 \pi}$ and $\sim 10^{-5}$ for $|z| \sim 1$. For $m=1$ and $n=40$, the relative error is $\sim 10^{-5}$ for $\left|z \pm \frac{i}{3 \pi}\right| \sim \frac{1}{3 . \pi}$ and $\sim 10^{-6}$ for $|z| \sim 1$. Accuracy is even better for a real argument, as shown in Figure 5, and differing from approximation (22), accuracy is also good in the oscillatory region. For a real argument and in the oscillatory region, $Q_{n}^{1}(n x) \simeq E_{n}(n x+1 / 2)$ and $P_{n}^{1}(n x)$ tends to zero exponentially fast. On the other hand, the approximation of $E_{n}(n x+1 / 2)$ in the monotonic region given by $P_{n}^{1}(n x)+Q_{n}^{1}(n x+1 / 2)$ is better than the approximation given by $P_{n}(n x)$. 


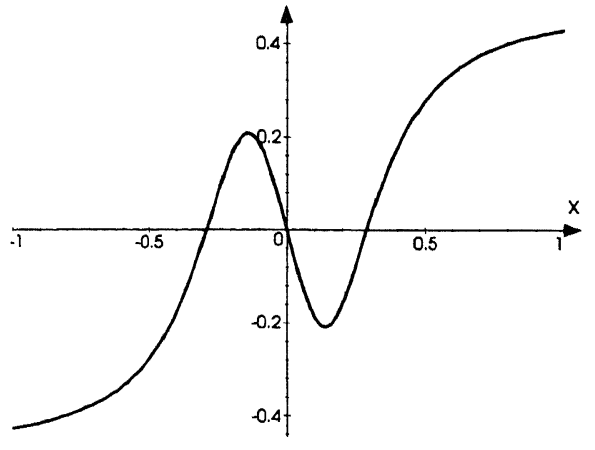

(a) $n=3$.

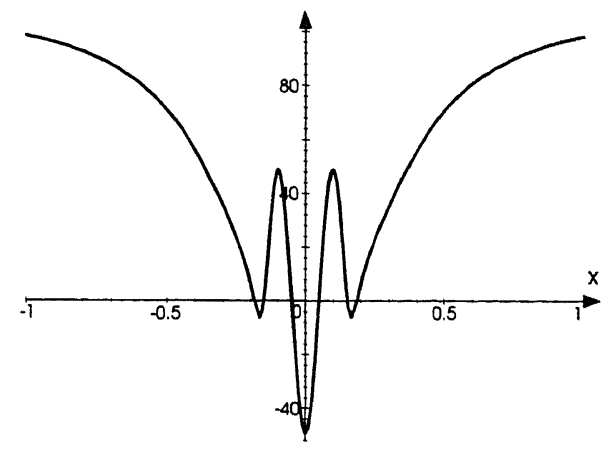

(b) $n=10$.

Figure 5. Solid lines represent $E_{n}(n x+1 / 2)$ for several values of $n$, whereas dashed lines represent the right-hand side of (35) with $z \equiv x$, both normalized by the same factor as in Figure 2.

\section{Conclusions}

Convergent expansions of the family of polynomials $P_{n}(n z)$ defined by formula (5) have been given in Equation (10). For the particular cases of Bernoulli $B_{n}(n z+1 / 2)$ and Euler $E_{n}(n z+1 / 2)$ polynomials, these expansions are given in Equations (19) and (22), respectively. They are uniformly valid for $\left|z^{-1}\right|<\left|z^{-1}-w_{i}\right| \forall i=1,2, \ldots$, where $w_{i}$ are the singularities of the meromorphic function $f(w)$ defining $P_{n}(n z)$ in (5) ordered by increasing modulus, $\left|w_{i}\right| \leq\left|w_{i+1}\right|$. For the particular cases of Bernoulli $B_{n}(n z+1 / 2)$ and Euler $E_{n}(n z+1 / 2)$ polynomials, convergence is restricted to the region $\left|z \pm \frac{i}{2 \pi}\right|>\frac{1}{2 \pi}$ and $\left|z \pm \frac{i}{\pi}\right|>\frac{1}{\pi}$, respectively, although the convergence is quite strong only outside the discs $|z| \leq \pi^{-1}$ and $|z| \leq 2 \pi^{-1}$, respectively, and slow inside these discs. Figures 2 and 3 show the accuracy of these approximations for several values of $n$ and a real argument $z$. Strong convergence is restricted to the monotonic region.

The convergence may be strongly accelerated by "isolating" the poles of $f(w)$, as described in Section 3. In this way we find that the convergence rate of expansion (10) is improved in expansion (29). In particular, the convergence rate of the expansions (19) and (22) of Bernoulli and Euler polynomials are improved by (32) and (35), respectively. These "improved" expansions are valid in a larger region of the uniformity parameter $z$. They are uniformly valid for $\left|z^{-1}\right|<\left|z^{-1}-w_{i}\right| \forall i=m+1, m+2, \ldots$ with $m$ a positive integer. In the particular cases of the Bernoulli and Euler polynomials, expansions (32) and (35) are uniformly valid for $\left|z \pm \frac{i}{2(m+1) \pi}\right|>\frac{1}{2(m+1) \pi}$ and $\left|z \pm \frac{i}{(2 m+1) \pi}\right|>\frac{1}{(2 m+1) \pi}$, respectively. For a real argument, the convergence is quite strong everywhere including the oscillatory region. Figures 4 and 5 
show the accuracy of these approximations for several values of $n$ and a real argument. We notice that the expansions in (32) and (35) are much better at the origin $z=0$ than the simpler expansions (19) and (22). This can be explained by observing that the latter expansions, though not correct, are exponentially small at the origin and that the contributions from the incomplete gamma functions can be viewed as first terms of fast converging expansions for the Bernoulli and Euler polynomials (see Remarks 2 and 3).

Approximations (19) and (22) may be quite useful for practical evaluations of Bernoulli $B_{n}(n z+1 / 2)$ and Euler $E_{n}(n z+1 / 2)$ polynomials for large $n$ in the appropriate region of $z$. When the uniformity parameter satisfies $|z|>$ $\pi^{-1}$ or $|z|>2 \pi^{-1}$, respectively, only the three first terms of the expansion (19) or (22) are needed to approximate $B_{10}(10 z+1 / 2)$ or $E_{10}(10 z+1 / 2)$ with two digits. For $|z|>1$, the first three terms approximate these polynomials with four digits. Because of the asymptotic character, the accuracy of the approximation increases for increasing $n$.

Only the first few terms of the expansion of $P_{n}^{1}(n z)$ are needed to obtain a good approximation of Bernoulli and Euler polynomials in a larger region of the uniformity parameter $z$ by using the improved approximations (32) and (35) containing incomplete gamma functions. Besides, the accuracy of these expansions is greater. On the other hand, the knowledge of the zeros of the incomplete gamma functions may be used to approximate the zeros of the Bernoulli and Euler polynomials by approximations (32) and (35).

\section{Acknowledgments}

J. L. López wants to thank C.W.I. of Amsterdam for its hospitality during the realization of this work. The financial support of C.I.C.Y.T., D.G.A. $(\mathrm{CONSI}+\mathrm{D})$ and CAI of Spain are acknowledged.

\section{References}

1. M. Abramowitz and I. A. Stegun, Handbook of Mathematical Functions, Dover, New York, 1970.

2. L. M. Milne-Thomson, The Calculus of Finite Differences, 2nd ed., Macmillan, New York, 1965.

3. C. Jordan, Calculus of Finite Differences, Chelsea, New York, 1947.

4. A. Fletcher, J. C. P. Miller, L. Rosenhead, and L. J. Comrie, An Index of Mathematical Tables, 2nd ed., Blackwell Science, Oxford, 1962.

5. A. Weinmann, Asymptotic expansions of generalized Bernoulli polynomials, Proc. Camb. Phil. Soc. 59:73-80 (1963).

6. P. G. Todorov, On the theory of the Bernoulli polynomials and numbers, J. Math. Anal. Appl. 104:309-350 (1984). 
7. K. Dilcher, Asymptotic behaviour of Bernoulli, Euler and generalized Bernoulli polynomials, J. Approx. Theor. 49:321-330 (1987).

8. K. Dilcher, Zeros of the Bernoulli, generalized Bernoulli and Euler polynomials, Mem. Amer. Math. Soc. 73(386):iv+94 (1988).

9. D. J. LeEmING, The real zeros of the Bernoulli polynomials, J. Approx. Theor. 58:124-150 (1989).

10. H. HARUKY and T. M. Rassias, New integral representations for Bernoulli and Euler polynomials, J. Math. Anal. Appl. 175:81-90 (1993).

11. D. CVIJovic and J. KLINOwSKI, New formulae for the Bernoulli and Euler polynomials at rational arguments, Proc. Amer. Math. Soc. 123(5):1527-1535 (1995).

12. A. Erdélyi, W. Magnus, F. Oberhettinger, and F. G. Tricomi, Higher Transcendental Functions, Vol 1. McGraw-Hill, New York, 1953.

13. W. Magnus, F. Obergettinger, and R. P. Soni, Formulas and Theorems for the Special Functions of Mathematical Physics, Springer-Verlag, Berlin, 1966.

14. I. S. GRADSHTEYN and I. M. RYZHIK, Table of Integrals, Series and Products, Academic Press, New York, 1980.

15. A. P. Prudnikov, Yu. A. Brychkov, and O. I. MARIChev, Integrals and Series, Gordon and Science, 1990.

16. N. M. TeMme, Special Functions: An Introduction to the Classical Functions of Mathematical Physics, Wiley, New York, 1996.

17. R. WONG, Asymptotic Approximations of Integrals, Academic Press, New York, 1989.

18. N. M. TEMME, Uniform asymptotic expansions of Laplace type integrals, Analysis 3:221249 (1983).

19. N. M. Temme, Laplace type integrals: Transformation to standard form and uniform asymptotic expansions, Quart. Appl. Math. 43:103-123 (1985).

20. N. M. TEMME, Uniform asymptotic expansions of a class of integrals in terms of modified Bessel functions, with application to confluent hypergeometric functions, SIAM. J. Math. Anal. 21:241-261 (1990).

21. N. M. Temme, Uniform asymptotic expansions of integrals: A selection of problems, $J$. Comp. Appl. Math. 65:395-417 (1995).

\section{UNIVERSIDAD DE ZARAGOZA, SPAIN \\ CWI, AMSTERDAM}

(Received September 8, 1998) 